\title{
On using Rayleigh scattering for in-situ spectral calibration of Thomson scattering diagnostics
}

\author{
S.A. Bozhenkov, ${ }^{1}$, a) S.J. Heym, ${ }^{2}$ M.N.A. Beurskens, ${ }^{1}$ G. Fuchert, ${ }^{1}$ E. Pasch, ${ }^{1}$ E.R. Scott,${ }^{1}$ and R.C. Wolf ${ }^{1}$ \\ 1) Max-Planck-Institut für Plasmaphysik, D-17491 Greifswald, Germany \\ ${ }^{2)}$ Faculty of Mathematics and Natural Sciences, Technical University of Berlin, Hardenbergstrasse 36, \\ D-10623 Berlin, Germany
}

A new method for in-situ spectral calibration of Thomson scattering diagnostics is proposed. The idea of the method is to apply a wavelength tunable optical parametric oscillator (OPO) for measurements of Rayleigh scattering at different wavelengths, from which relative sensitivities can be calculated. This extends the usual approach, where Rayleigh scattering is used only at a single wavelength for the absolute calibration and spectral sensitivities are obtained separately. With the new approach, the full diagnostic setup is spectrally calibrated at once. Such a calibration can be repeated at regular intervals during an experimental campaign, since it does not require a break of the vacuum. In this paper, the Rayleigh scattering calibration is tested in a laboratory setup with a sample Wendelstein 7-X (W7-X) polychromator. It is shown that relative sensitivities of spectral channels can be recovered with a sufficient resolution even under conditions of significant stray light. The stray light is overcome by measuring the linear dependence of the scattered signal on the gas pressure. Good results of laboratory tests motivate the installation of the new calibration system for the Thomson scattering diagnostic at W7-X.

\section{INTRODUCTION}

Thomson scattering ${ }^{1}$ is a standard diagnostic at many fusion experiments ${ }^{2-6}$. In particular, a full profile Thomson scattering system is installed at the large optimized stellarator Wendelstein $7-\mathrm{X}(\mathrm{W} 7-\mathrm{X})^{7,8}$. Three Nd:YAG lasers, up to $2.4 \mathrm{~J}$ at $10 \mathrm{~Hz}$ each, are used to give an effective measurement frequency of $30 \mathrm{~Hz}$. Presently, 42 positions along the laser beams passing horizontally through the plasma are observed with two collection optics. A further extension to 95 scattering volumes is foreseen. The diagnostic is routinely operated to provide time resolved profiles of both the electron density and electron temperature. Such profiles are extensively applied for transport and stability studies and as an input for various simulations.

The quality of the resulting temperature and density profiles depends on the diagnostic calibration procedure. Subtle differences in calibration and measurement conditions may result in systematic errors visible both in the temperature and in the density. For example, measurements with Ge-doped fibers having a larger numerical aperture of 0.37 , as compared to 0.29 for quartz fibers, showed systematic discrepancies in the temperature during the first experimental campaign of $\mathrm{W} 7-\mathrm{X}^{8}$. Systematic errors in the density can be observed also in later results from $\mathrm{W} 7-\mathrm{X}^{9}$.

The present calibration scheme of the W7-X Thomson scattering diagnostic consists of two steps ${ }^{8}$. The relative spectral calibration of five polychromator channels is carried out with the help of a supercontinuum light source (Super-K compact by NKT Photonics) and a scanning monochromator. The monochromator output transferred via a fiber bundle is used to illuminate a diffuse screen in front of the Thomson collection optics.

${ }^{a)}$ Electronic mail: sergey.bozhenkov@ipp.mpg.de
Relative sensitivities of the channels are obtained by a consecutive scan of the wavelength selected with the monochromator. The absolute density calibration is performed by anti-Stokes rotational Raman scattering in nitrogen. The Raman procedure is characterized by a high statistical precision of better than $1 \%$. However, systematic errors in the density are much larger, because only one, or in the best case two low temperature channels, are calibrated directly. Uncertainties in the spectral calibration propagate also into the density for typical plasma temperatures.

The described spectral calibration procedure has several potential drawbacks. Firstly, the front vacuum window is not a part of the spectral calibration. The window can be potentially covered during plasma operation and boronization of the first wall, which changes its transmission in a wavelength dependent manner. Secondly, it is hard to establish an equivalent fiber filling from the diffuse screen. As a result, relative sensitivities of the channels can be offset due to the illumination conditions. In fact, this is a possible explanation for the aforementioned systematic problems with Ge-fibers, because they are especially sensitive to the illumination conditions due to their large numerical aperture. In addition, the Raman scattering calibration requires a very high accuracy of the spectral calibration, since Raman lines are partially located at the sharp edges of the filters ${ }^{8}$.

The need to improve calibration of Thomson scattering diagnostics is not unique to $\mathrm{W} 7-\mathrm{X}$ and is also recognized at other experiments. An in-vessel calibration with the help of an integrating sphere seems appropriate, as it removes both problems. But, the vessel access is often limited or even hardly possible. In addition, integrating spheres are often operated in CW-mode, which requires extra measurement electronics. These limitations can be partially avoided by using a pulsed light source and an insertable integrating sphere ${ }^{10}$. Though, this method includes only a few wavelengths, 


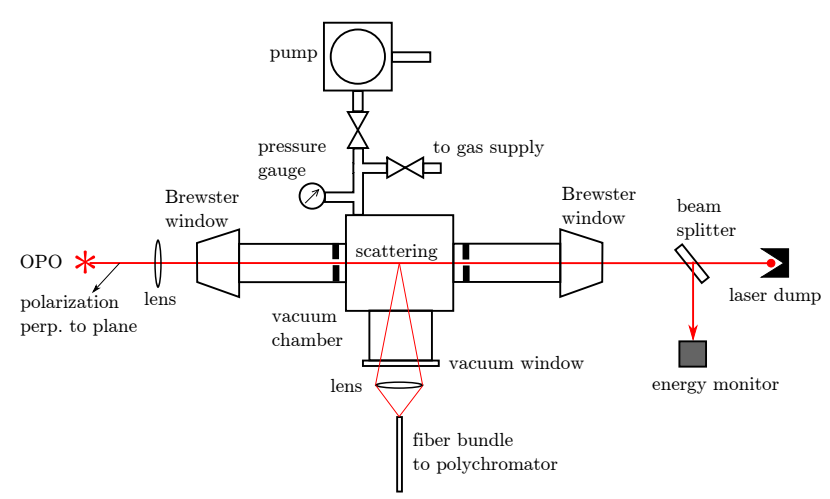

FIG. 1. Experimental setup for testing the Raleigh scattering calibration. The linearly polarized OPO is focused into the center of the vacuum chamber. The scattered light is observed with a collection lens, a fiber bundle and a polychormator. The vacuum chamber can be pumped down and filled with a test gas to a desired pressure via a needle valve. Further components of the setup are: focusing lens, Brewster windows, limiting apertures for stray light reduction, laser beam dump and laser energy monitor. The inside of the vacuum chamber is covered with a diffuse low-reflectance foil.

requires movable vacuum parts, and, probably therefore, is not adopted widely. Instead, a lot of interest was shown recently for in-situ spectral calibration combined with plasma measurements. It can be achieved either by observing the same scattering volume from two angles, as realized in a double pass system ${ }^{11,12}$, or alternatively, the same effect can be obtained with a dual wavelength system $^{13,14}$. A dual wavelength system is considered for ITER $^{15,16}$ and is also under development for $\mathrm{W} 7-\mathrm{X}^{17}$.

Here, we propose another method for spectral calibration of Thomson scattering diagnostics. In this method, an optical parametric oscillator (OPO) of sufficient power is guided as close as possible to the measurement laser and is used to measure Rayleigh scattering for the full range of wavelengths. In such a way all components of the diagnostic, including the vacuum window and the measurement electronics, are taken into account. The uncertainty caused by the illumination conditions is removed, too, because the angular distribution of the scattered light is close to plasma measurements. The calibration does not require vessel access nor vacuum movable parts. It is sufficient to fill the vacuum vessel with an inert gas, e.g. nitrogen or argon, which can be effectively combined with the absolute calibration of the Thomson system and can be repeated regularly.

It is to be noted that a similar method was suggested before $^{18}$. A dye laser with four different dyes was proposed for Rayleigh scattering to cover the spectral range from 680 to $840 \mathrm{~nm}$. Unfortunately, to our knowledge experimental results of such a calibration were never published. The use of OPO that can nowadays generate required powers simplifies the setup and potentially allows to cover the spectral range from $410 \mathrm{~nm}$ to $2500 \mathrm{~nm}$. Here we consider a narrower range from 700 to $1100 \mathrm{~nm}$ suitable for the W7-X Thomson scattering system.

In this paper, the Rayleigh scattering calibration is

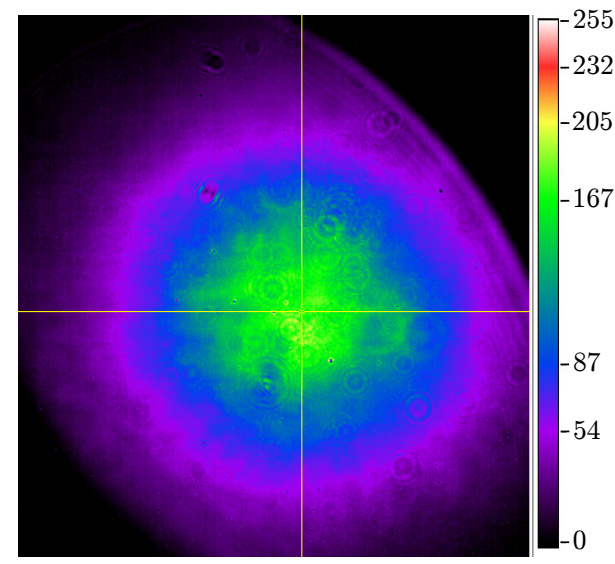

FIG. 2. Beam profile measured with a camera at the distance of $6 \mathrm{~m}$ from the OPO output. The beam size is about $1 \mathrm{~cm}$ in both directions.

tested in a dedicated laboratory setup with a sample W7-X polychromator. It is demonstrated for the first time that the available commercial OPO is sufficient to obtain relative sensitivities, even if a large stray light background is present. The active signal is discriminated by using the linear dependence of Rayleigh scattering on the gas pressure. Two gasses, nitrogen and argon, are compared and found to give similar results. The spectral resolution of the OPO system is sufficiently good, though minor differences to the standard supercontinuum calibration can be see on sharp edges.

\section{EXPERIMENTAL SETUP}

The laboratory setup for testing the Rayleigh scattering calibration is presented in fig. 1 . It is based on a cylindrical vacuum chamber of about $38 \mathrm{~cm}$ in diameter and about $24 \mathrm{~cm}$ in height. An observation window, about $12 \mathrm{~cm}$ in diameter, is installed at the front of the chamber. At the backside the chamber is connected to a vacuum system and to a gas supply. Two tubes of a typical diameter of $10 \mathrm{~cm}$ and about $30 \mathrm{~cm}$ in length are connected to the sides of the cylinder. They serve as the input and output ports for the OPO beam. The linearly polarized OPO beam is focused with a lens with a focal length of $2 \mathrm{~m}$ into the center of the volume. After the output window the beam is dumped into a conical laser dump at sufficient distance. The energy of OPO pulses is monitored with a pyroelectric detector PEM 45K USB (Sensor und Lasertechnik $\mathrm{GmbH}$ ) having negligible spectral dependence in the wavelength region of interest.

The OPO (Spitlight 1000 by InnoLas Laser) is pumped by a frequency doubled $10 \mathrm{~Hz} \mathrm{Nd}$ :YAG laser and can be operated in the wavelength range from 670 to $1100 \mathrm{~nm}$. The output light pulses are about $10 \mathrm{~ns}$ long with the same frequency of $10 \mathrm{~Hz}$, i.e. their timing is very similar to that of the Nd:YAG lasers used for the Thomson scattering diagnostic. The output energy varies with the wavelength, with the maximum being about $150 \mathrm{~mJ}$ at 


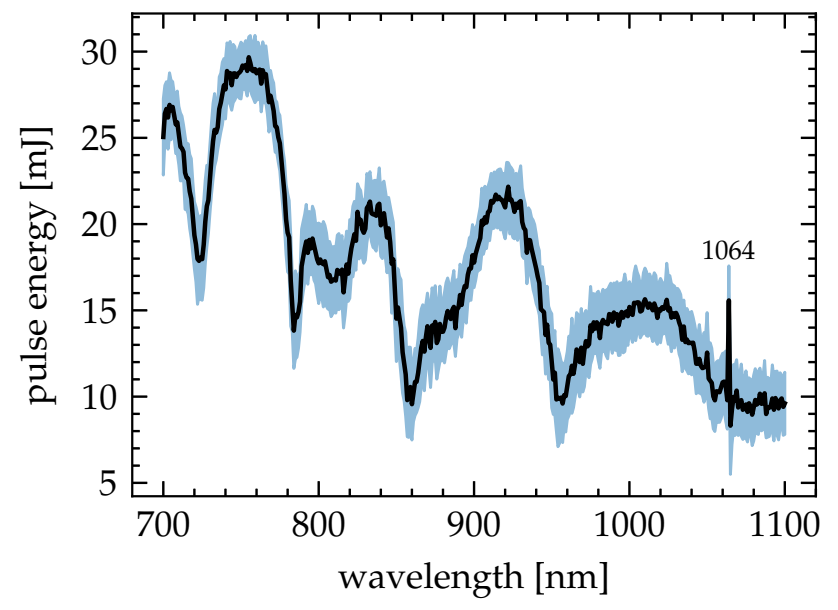

FIG. 3. Dependence of the OPO pulse energy on the wavelength. The black line is an average of 40 pulses for each wavelength. The light blue area shows a typical pulse to pulse variation. The OPO was used with the reduced energy.

$750 \mathrm{~nm}$. The OPO line width increases with the wavelength: about $0.2 \mathrm{~nm}$ at $750 \mathrm{~nm}$, about $0.6 \mathrm{~nm}$ at $1000 \mathrm{~nm}$ and about $0.8 \mathrm{~nm}$ at $1100 \mathrm{~nm}$. The OPO beam is about $1 \mathrm{~cm}$ in diameter and its typical divergence is about $2 \mathrm{mrad}$. An example of the beam profile at the distance of $6 \mathrm{~m}$ measured with a camera is given in fig. 2 . It is to be noted that such a high profile quality was achieved after an upgrade by the manufacturer and the original measurements were performed with a more elliptical beam.

Scattered light is collected with a short focus achromatic lens into a fiber bundle with numerical aperture of 0.29 . The other end of the bundle is attached to a standard W7-X polychromator with five spectral channels ${ }^{8}$. Spectral ranges covered by the channels are $750-920 \mathrm{~nm}$, 920-1000 nm, 1000-1035 nm, 1035-1051 nm and 1051$1061 \mathrm{~nm}$. The signals are measured with avalanche photodiodes and digitized with fast data acquisition boards at $1 \mathrm{GS} / \mathrm{s}$ and 14 bit resolution (ADQ-14 by SP Devices). The polychromator, photodiodes and the acquisition system are the same as used for the W7-X Thomson scattering system.

Several measures were taken in order to reduce stray light to a tolerable level. The input and output vacuum windows are installed at the Brewster angle. Two limiting apertures of $2 \mathrm{~cm}$ in diameter are inserted into the input and output ports, as shown in fig. 1. The inner surface of the vacuum chamber is covered with a low reflectance diffuse foil with a specular reflection coefficient below $0.02 \%$ (MetalVelvet by Acktar). In addition, a polarizer is used in front of the observation fiber bundle. Despite these measures, significant stray light is still present in the measurements. In this paper it is demonstrated that a reliable spectral calibration is nevertheless possible. The scattered signal is discriminated by a pressure scan, similar to absolute calibrations with Rayleigh scattering.

Both the OPO and the data acquisition are controlled programmatically. The wavelength of the OPO can be chosen remotely via a TCP/IP connection. This will allow an easy integration into the W7-X control system and

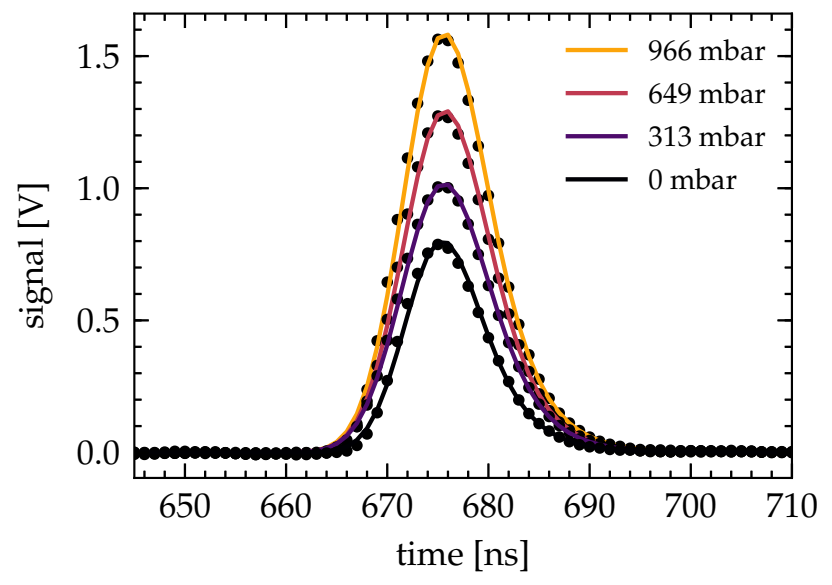

FIG. 4. Raw signals for several pressure values in Ar. These examples are for channel 1 at $\lambda=921 \mathrm{~nm}$. The stray light level is evident from the vacuum signal. The change of the signal with the gas pressure is due to the addition of Rayleigh scattering.

simplifies wavelength scans. In this work, two types of wavelength scans were performed for each pressure value. The first one is a full wavelength scan between 700 and $1100 \mathrm{~nm}$ with the step of $1 \mathrm{~nm}$. The second one covers only the spectral channel 5 between 1047 and $1062 \mathrm{~nm}$ but with the wavelength step of $0.1 \mathrm{~nm}$. It is worth noting that the step of $0.1 \mathrm{~nm}$ is below the OPO line width. In both cases ten laser pulses were measured per wavelength. It takes less than 15 minutes to complete a single full wavelength scan.

\section{EXPERIMENTAL RESULTS}

The output pulse energy of the OPO varies strongly with the wavelength. The wavelength dependence measured with the pyroelectric detector in a series of 40 pulses per wavelength is shown in fig. 3 . The pulse to pulse variation of the energy at each wavelength is indicated in the same figure with a colour band. It is obvious that for reliable calibration the OPO energy should be recorded and preferably for each pulse in the case of a restricted number of pulses. For the present experiments the OPO was used with a reduced pulse energy to avoid signal saturation by stray light.

Raw signals for one of the spectral channels and for different argon pressures are presented in fig. 4. The experimental data points are fitted with a convolution of a Gaussian peak with the first order low-pass filter. Time constants of the Gaussian pulse and of the low-pass filter are free fit parameters. As the main interest is in the pulse integral, no particular attention was paid to the refinement of time constants. The chosen pulse shape is sufficient for current purposes.

A significant level of stray light is evident from the high signal amplitude in the vacuum run. Given the small size of the test setup, it is not possible to distinguish the stray light and the scattered signal from changes of the pulse shape. Instead, the Rayleigh part can be recognized in a 


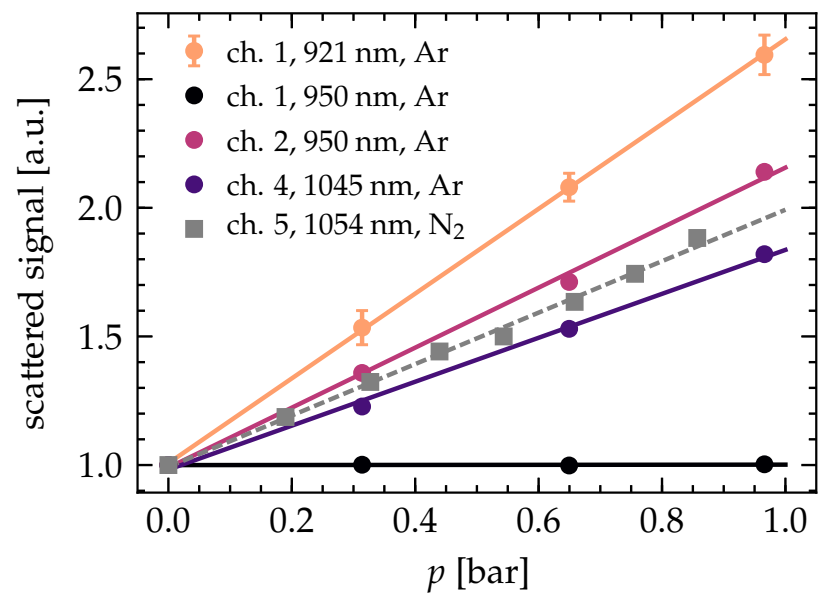

FIG. 5. Scaling of the scattered signal with the gas pressure. The dependence is given for channel 1 at wavelengths of 921 and $950 \mathrm{~nm}$, channel 2 at wavelength of $950 \mathrm{~nm}$ and channel 4 at wavelength of $1045 \mathrm{~nm}$, as measured in Ar. For comparison results for channel 5 at wavelength of $1054 \mathrm{~nm}$ measured in nitrogen are also shown. An arbitrary offset is added to each line for clarity. Standard deviations estimated from ten laser pulses are shown for one of the cases.

pressure scan, because it scales linearly with the pressure, whereas the stray light is independent of the pressure.

To illustrate separation of the scattered signal it is appropriate to write an expression for the photodiode signal. The integral signal registered in the spectral channel $i$ during an OPO pulse can be formulated as:

$$
\left(\int s d t\right)_{i}=b(\lambda) \cdot \varepsilon(\lambda)+n_{\text {gas }} \cdot \varepsilon(\lambda) \cdot \frac{d \sigma}{d \Omega} \cdot \frac{\lambda}{h c} \frac{g_{i}(\lambda)}{g_{0}} \cdot \delta L \delta \Omega g_{0}
$$

Here $b(\lambda)$ is a combined factor for conversion and measurement of the stray light, $\varepsilon(\lambda)$ is the OPO pulse energy, $n_{\text {gas }}$ is the gas particle density, $d \sigma / d \Omega$ is the differential scattering cross-section, $g_{i}(\lambda) / g_{0}$ is the relative spectral calibration factor normalized to photons, and $\delta L \delta \Omega g_{0}$ is the absolute calibration factor consisting of the scattering volume length, observation solid angle and the absolute sensitivity of the reference channel. The first term on the right hand side describes the stray light contribution, which is assumed to be proportional to the input energy and to depend on the wavelength, but not on the pressure. The second term is the Rayleigh scattered part. The signal normalized by the pulse energy scales linearly with the pressure, with the linear slope containing only physical constants and observation system parameters. This is fully analogous to the absolute calibration by Rayleigh scattering, albeit here the spectral sensitivity factor $g_{i}(\lambda) / g_{0}$ is considered unknown and the absolute factor is ignorable.

Linear dependence of the signal on the gas pressure is confirmed experimentally. The measured signal normalized by the OPO pulse energy is shown in fig. 5 for several chosen wavelengths and chosen spectral channels. The data set includes four pressure points obtained in argon and, for comparison, eight pressure points obtained in nitrogen. A clear linear trend is found in all cases. The

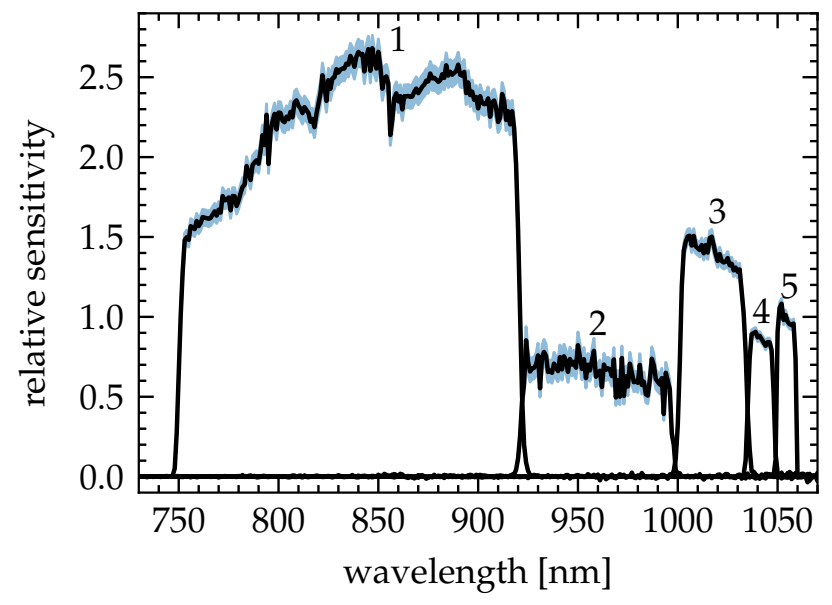

FIG. 6. Relative sensitivities of five spectral channels measured from Rayleigh scattering in Ar. They are calculated from linear slopes of the Rayleigh signal scaling with pressure. The sensitivities are normalized to that of channel 5 at $1054 \mathrm{~nm}$. The coloured patches represent an estimation of the statistical error range.

typical statistical error is about $3 \%$ for all channels but channel 2. For the latter case the error is about $10 \%$ due to higher noise in the used photodiode.

The slope of lines in fig. 5 is proportional to the product of the scattering cross-section and of the relative sensitivity. Relative sensitivities can be calculated from linear slopes of the pressure scaling, provided the wavelength dependence of the scattering cross-section is known. Other factors in the slope coefficient are the same for all spectral channels and are removed by normalization. For simplicity, we assume the Rayleigh scattering crosssection to be inversely proportional to the forth power of the wavelength: $\sigma \sim 1 / \lambda^{4}$. The used approximation is accurate within about $1-2 \%^{19-22}$. In general, the proposed calibration method is not limited to the simplified cross-section form.

Relative sensitivities deduced from the measurements at four pressures in argon with ten OPO pulses per wavelength are shown in fig. 6 . The sensitivities are normalized to that of the fifth spectral channel at $1054 \mathrm{~nm}$. The overall shapes of sensitivities agree well with the standard calibration method ${ }^{8}$. The five spectral channels are located in the expected wavelength ranges. Outside of the sensitivity ranges the results exhibit typical signal level between $10^{-3}-10^{-2}$ depending on the photodiode noise. Thus, the dynamic range of the present method can be as good as 1000 . The uncertainty range of the spectral sensitivity estimated from measurement errors is also shown in figure 6 . The final error is typically in the range from 3 to $5 \%$ for all channels with exception of channel 2 . In the latter case the error is about $10 \%$. It is worth noting that the error can also be concluded from the point to point variation of the spectral sensitivity.

Residual oscillations of about $10 \%$, i.e. above the statistical uncertainty, can be observed in channel 1 between 800 and $900 \mathrm{~nm}$ in fig. 6 . The nature of the wave pattern is not understood. It can be either actually present 
or, more likely, can be caused by measurement imperfections, e.g. by details of the present optical setup, by errors in energy measurements, or by nonlinearity of the photodiode close to saturation. An in-depth analysis will be performed in future, once both the test polychromator and the new calibration method are installed at W7-X, where the standard calibration method can be applied for a direct comparison. A higher noise level in channel 2 is caused by characteristics of the avalanche photodiode. Similar channel sensitivities were obtained also in nitrogen. For example, in fig. 7a results for channel 5 from scattering in argon and nitrogen are overlayed. The general sensitivity shape agrees within the measurement errors between both gases. However, there are indications that channel edges obtained in nitrogen are extended by the rotational Raman scattering at the percent level in the range from 5 to $10 \mathrm{~nm}$, as can indeed be expected from the relative level of the Raman cross-section ${ }^{8,19}$. A further reduction of the noise level, e.g by increasing the number of used laser pulses, is required to investigate the Raman effect quantitatively. Nevertheless, it can be concluded that calibration in nitrogen can be conveniently used to estimate relative sensitivities for Thomson scattering measurements. But, a more precise calibration of the edges in argon may be required to apply the data for the absolute Raman calibration.

To examine the spectral resolution of the method in argon, a finer wavelength scan with the step size of $0.1 \mathrm{~nm}$ was conducted for channel 5 . Channel 5 is chosen for the comparison, because the Rayleigh signal is the lowest at long wavelengths due to both reduced scattering crosssection and reduced OPO energy. It is to be noted that channel 5 is a reference channel, and, hence, the amplitude for all measurements is the same. The channel 5 sensitivity from the detailed scan is compared in fig. $7 \mathrm{~b}$ with the outcome of the $1 \mathrm{~nm}$ scan and in fig. $7 \mathrm{c}$ with the typical shape obtained with the standard calibration method. Results of the detailed scan and of the standard calibration are in good agreement. The latter appears smoother due to better statistics: typically a few hundred supercontinuum light pulses, as compared to forty summed over all pressures in the present study, are collected in such measurements. A subtle difference can be seen on the channel edges, which can be explained by a coarser spectral resolution provided by the OPO. The OPO line width is about $0.8 \mathrm{~nm}$ in this wavelength range, whereas the standard method has spectral resolution of better than $0.5 \mathrm{~nm}$ defined by the monochromator ${ }^{8}$. A further reduction of the OPO line width is under discussion with the manufacturer. Nevetheless, the Rayleigh scattering calibration is undoubtedly applicable for Thomson measurements and can be used also for the absolute Raman calibration for comparison.

\section{SUMMARY}

A new method for in-situ spectral calibration of Thomson scattering diagnostics is proposed. In this method, a wavelength tunable OPO is guided as close

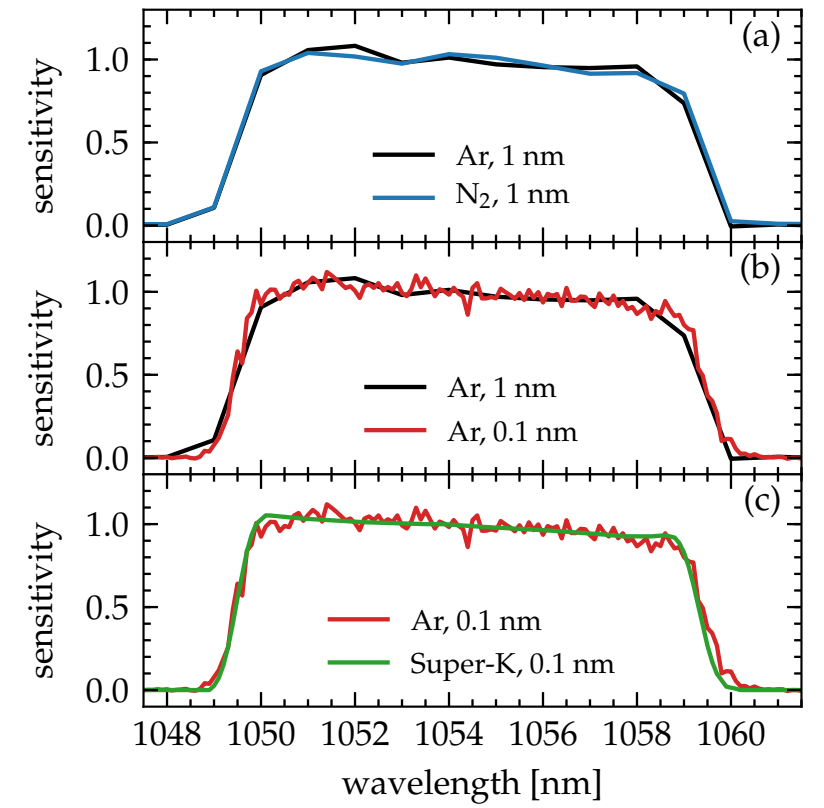

FIG. 7. Comparison of the sensitivity for channel 5 measured in different ways. (a) - comparison of the Rayleigh scattering measurements in argon and nitrogen with the wavelength step of $1 \mathrm{~nm}$. (b) - comparison of the Rayleigh scattering measurements in argon with the wavelength step of 1 and $0.1 \mathrm{~nm}$. (c) - comparison of the Rayleigh scattering measurements with the standard calibration procedure. The wavelength step is $0.1 \mathrm{~nm}$ for both methods, but the number of used laser pulses is 10 for the Rayleigh scattering and 500 for the calibration with the supercontinuum source.

as possible to the measurement laser, and Rayleigh scattering at different wavelengths is used to deduce relative sensitivities. The full diagnostic setup, including the vacuum window and the measurement electronics, is calibrated at once. The angular distribution of Rayleigh scattered light approximates well that of Thomson measurements and, thus, the uncertainty due to the illumination conditions is also minimized. The new method does not require a break of the vacuum and can be repeated at regular intervals in a combination with the absolute density calibration. All spatial points of the diagnostic can be calibrated simultaneously, which reduces the required calibration time.

The Rayleigh scattering calibration in argon and nitrogen was tested in a dedicated laboratory setup with a sample W7-X polychromator. It is shown that relative sensitivities can be obtained even with significant stray light by measuring the linear dependence on the gas pressure. Scanning the pressure also helps to compensate the strong reduction, by a factor of four in the wavelength range of interest, of the Rayleigh scattering cross-section at long wavelengths. In general, the obtained calibration curves agree well with the expectations and previous experience. Residual oscillations are observed in one of the channels, which can be potentially related to the details of the laboratory optical setup, to the non-linearity of the used APD close to the full amplitude or to systematic errors in the energy measurements. 
Further studies of this effect will be reported separately, once the new calibration scheme becomes available at W7-X. Apart from that, the new method is able to provide a good spectral resolution even at the edges, as limited only by the OPO line width. A further reduction of the OPO line width is under discussion with the manufacturer. It is the first experimental demonstration that Rayleigh scattering is applicable for the spectral calibration of Thomson scattering diagnostics.

Good results of the laboratory tests motivate the installation of the new calibration system for the W7-X Thomson scattering diagnostic. The installation is work in progress and its results will be reported separately. The major uncertainty is the yet unknown level of stray light. Although, it is believed to become less critical because of a much larger size of the system, e.g. the input and output ports are about 3.5 and $2.4 \mathrm{~m}$ long respectively, and the typical size of the vacuum vessel is about $1.5 \mathrm{~m}$. In addition, a corrugated channel for the stray light reduction is installed in the entrance port. If stray light proves to be tolerable, the spectral Rayleigh calibration can be performed at typical pressures of 100 mbar, which is in the range used for the absolute Raman calibration. The accessible pressure range follows both from the available reserve in the OPO energy and from the comparison of the Rayleigh and Raman cross-sections. Experiments with the spectral Rayleigh calibration at W7-X will allow a direct comparison with the standard calibration procedure and with the double wavelength calibration. The use of the proposed method as a cross check of the absolute Raman calibration will also be considered.

There are at least two further points concerning the new method. Firstly, the OPO beam quality is usually worse than that of standard Nd:YAG lasers. However, this is not believed to be a limiting factor, as long as the focused beam can be assured to lie inside the volume observed by the collection optics and fiber bundles. It is possible to intentionally verify the sensitivity of the spectral calibration to the beam position. If large variations are found, it may indicate a need for the polychromator readjustment, since the position of the measurement laser is also controlled with a limited precision. Secondly, it can be argued that the input and output vacuum windows for the OPO are covered by plasma depositions as well. But in contrast to the observation window, these windows, at least at W7-X, are far away from the plasma at the end of narrow ports. In addition, they are probably cleaned either by the OPO or Nd:YAG radiation. The latter assumption can be verified experimentally by observing the OPO pulse energy before and after the vacuum vessel.

\section{ACKNOWLEDGMENTS}

Authors would like to thank Dr. J. Baldzuhn, Dr. C. Biedermann and Dr. M. Hirsch for technical support.

This work has been carried out within the framework of the EUROfusion Consortium and has received funding from the Euratom research and training programme 2014-2018 under grant agreement No 633053. The views and opinions expressed herein do not necessarily reflect those of the European Commission.

${ }^{1}$ I. Hutchinson, Principles of Plasma Diagnostics (Cambridge University Press, 2005).

${ }^{2}$ R. Pasqualotto, P. Nielsen, C. Gowers, M. Beurskens, M. Kempenaars, T. Carlstrom, D. Johnson, and J.-E. Contributors, Review of Scientific Instruments 75, 3891 (2004), http://dx.doi.org/10.1063/1.1787922.

${ }^{3}$ B. Kurzan and H. D. Murmann, Review of Scientific Instruments 82, 103501 (2011), http://dx.doi.org/10.1063/1.3643771.

${ }^{4}$ I. Yamada, K. Narihara, H. Funaba, R. Yasuhara, T. Kohmoto, H. Hayashi, T. Hatae, H. Tojo, T. Sakuma, H. Yoshida, H. Fujita, and M. Nakatsuka, Journal of Instrumentation 7, C05007 (2012).

${ }^{5}$ T. N. Carlstrom, G. L. Campbell, J. C. DeBoo, R. Evanko, J. Evans, C. M. Greenfield, J. Haskovec, C. L. Hsieh, E. McKee, R. T. Snider, R. Stockdale, P. K. Trost, and M. P. Thomas, Review of Scientific Instruments 63, 4901 (1992), http://dx.doi.org/10.1063/1.1143545.

${ }^{6}$ J. W. Hughes, D. Mossessian, K. Zhurovich, M. DeMaria, K. Jensen, and A. Hubbard, Review of Scientific Instruments 74, 1667 (2003), http://dx.doi.org/10.1063/1.1532764.

${ }^{7}$ E. Pasch, M. N. A. Beurskens, S. A. Bozhenkov, G. Fuchert, J. Knauer, and R. C. Wolf, Review of Scientific Instruments 87, 11E729 (2016), http://aip.scitation.org/doi/pdf/10.1063/1.4962248.

${ }^{8}$ S. Bozhenkov, M. Beurskens, A. D. Molin, G. Fuchert, E. Pasch, M. Stoneking, M. Hirsch, U. Höfel, J. Knauer, J. Svensson, H. T. Mora, and R. Wolf, Journal of Instrumentation 12, P10004 (2017).

${ }^{9}$ R. Wolf and et al., submitted to Plasma Physics and Controlled Fusion (2018).

${ }^{10}$ H. D. Stephens, M. T. Borchardt, D. J. Den Hartog, A. F. Falkowski, D. J. Holly, R. OConnell, and J. A. Reusch, Review of Scientific Instruments 79, 10E734 (2008), https://doi.org/10.1063/1.2964229.

${ }^{11}$ H. Tojo, A. Ejiri, J. Hiratsuka, T. Yamaguchi, Y. Takase, K. Itami, and T. Hatae, Review of Scientific Instruments 83, 023507 (2012), https://doi.org/10.1063/1.3685612.

${ }^{12} \mathrm{H}$. Tojo, T. Hatae, and K. Itami, Journal of Instrumentation $\mathbf{7}$, C05004 (2012).

${ }^{13}$ O. R. P. Smith, C. Gowers, P. Nielsen, and H. Salzmann, Review of Scientific Instruments 68, 725 (1997), https://doi.org/10.1063/1.1147686.

${ }^{14}$ O. McCormack, L. Giudicotti, A. Fassina, and R. Pasqualotto, Plasma Physics and Controlled Fusion 59, 055021 (2017).

${ }^{15}$ R. Pasqualotto, L. Giudicotti, A. Alfier, and M. J. Walsh, Journal of Physics: Conference Series 227, 012044 (2010).

${ }^{16}$ L. Giudicotti and R. Pasqualotto, Nuclear Fusion 54, 043005 (2014).

${ }^{17}$ E. Pasch, M. N. A. Beurskens, S. A. Bozhenkov, G. Fuchert, and R. C. Wolf, Review of Scientific Instruments 89, $10 \mathrm{C} 115$ (2018), https://doi.org/10.1063/1.5038422.

${ }^{18}$ D. A. Rasmussen, R. R. Kindsfather, C. E. Thomas, R. P. Gormley, and S. L. Painter, Review of Scientific Instruments 59, 1464 (1988).

${ }^{19}$ R. B. Miles, W. R. Lempert, and J. N. Forkey, Measurement Science and Technology 12, R33 (2001).

${ }^{20}$ E. R. Peck and D. J. Fisher, J. Opt. Soc. Am. 54, 1362 (1964).

${ }^{21}$ E. R. Peck and B. N. Khanna, J. Opt. Soc. Am. 56, 1059 (1966).

${ }^{22}$ D. Bates, Planetary and Space Science 32, 785 (1984). 